\section{Arritmias cardíacas e parada cardiorrespiratória na gestação}

\section{1 - Arritmias cardíacas}

\section{Hélio Germinani}

Na gestação, as alterações hormonais e hemodinâmicas fisiológicas favorecem o surgimento de sintomas que sugerem a presença de arritmias. Palpitação é uma queixa frequente e geralmente está relacionada à taquicardia sinusal ${ }^{1,2}$. A investigação não invasiva é a mesma que em não gestantes ${ }^{2} \mathrm{e}$ as arritmias geralmente são documentadas entre $10-24 \%$ das pacientes sintomáticas, sendo que a maioria delas é benigna-4 .

A arritmia complica cerca de $6 \%$ das gestantes portadoras de cardiopatia, elevando para $17 \%$ naquelas com cardiopatia reumática ${ }^{5}$.

Pacientes com taquiarritmias prévias à gestação apresentam risco aumentado de eventos cardíacos, como a recorrência de arritmias (em 44\% e mesmo naquelas submetidas à ablação prévia ou em uso de antiarrítmicos), AVC, EAP e morte, como também eventos adversos fetais em 20\% (prematuridade, baixo peso ao nascer, disfunção respiratória, hemorragia intraventricular, morte) $)^{6}$.

\subsection{1 - Arritmias supraventriculares e ventriculares}

As arritmias cardíacas podem se manifestar como extrassistolia atrial, juncional ou ventricular e, eventualmente, como taquicardia paroxística supraventricular de curta duração ou ventricular, tanto em mulheres normais como em cardiopatas ${ }^{1-4}$.

Para estratificação de risco é fundamental a obtenção de um ecocardiograma, que possibilitará o diagnóstico de cardiopatia estrutural, além da avaliação da função ventricular. É necessária realização de provas de função tireoidiana para detectar ou descartar hipertireoidismo. Gestantes normais e assintomáticas que apresentam arritmias sem repercussão hemodinâmica não necessitam ser medicadas².

Gestantes cardiopatas apresentam com mais frequência arritmias supraventriculares, como extrassistolia atrial, fibrilação atrial ou flutter atrial. Entre as cardiopatias, as que mais se associam a essas arritmias são as que apresentam comprometimento atrial, como doença mitral reumática ou defeito do septo interatrial ${ }^{4}$.

A taquicardia paroxística por reentrada nodal é a mais frequente em mulheres, ocorrendo na ordem de $75 \%$ dos casos, sejam gestantes ou não ${ }^{7,8}$.

A taquicardia atrial primária é rara na gestação e geralmente se apresenta em gestantes sem doença cardíaca estrutural. Às vezes é necessário o controle da frequência cardíaca para evitar cardiomiopatia induzida por taquiarritmia. Pode necessitar do uso de bloqueadores beta-adrenérgicos ou até de amiodarona ${ }^{2}$.

Em mulheres portadoras de cardiomiopatia dilatada, a ocorrência de arritmias ventriculares, seja sob a forma de extrassístoles ou taquicardia fascicular, é muito comum e há necessidade de tratamento ${ }^{9,10}$.
A síndrome de Wolff-Parkinson-White não é rara durante a gestação, ocorrendo habitualmente em mulheres cardiologicamente normais. Se forem assintomáticas, não há necessidade do uso de medicação, podendo ser encaminhadas para ablação elétrica do feixe anômalo após o parto. Se, eventualmente, apresentarem crises recorrentes e incapacitantes de taquicardia paroxística supraventricular e sem resposta terapêutica, poderão, mesmo no decorrer da gestação, ser submetidas à ablação com radiofrequência, com o cuidado de se proteger o abdome e o dorso da gestante com avental de chumbo, evitando excesso de radiação para o feto ${ }^{11}$. O mesmo procedimento pode ser realizado em gestantes portadoras de taquiarritmias supraventriculares que não respondam a drogas antiarrítmicas ${ }^{12}$.

Pacientes portadoras da síndrome do QT longo (QTC > $470 \mathrm{~ms}$ ) apresentam redução de eventos cardíacos durante a gestação (síncope, PCR revertida, morte) e aumento no período de puerpério tardio (até 9 meses após o parto), acometendo mais as portadoras do genótipo tipo II. O uso de betabloqueadores beneficia essas pacientes, principalmente no puerpério ${ }^{13}$.

\subsection{2 - Transtornos da condução atrioventricular}

Gestantes que apresentem bloqueio A-V total congênito, com frequência ventricular entre 50-60 batimentos por minuto e assintomáticas toleram bem a gestação, sem necessidade de implante de marca-passo artificial. As portadoras de bloqueio A-V de segundo grau tipo II, de bloqueio A-V total e que necessitam receber implante de marca-passo artificial poderão recebê-lo, desde que durante o procedimento se proteja o abdome e o dorso com avental de chumbo ${ }^{14,15}$. É sugerido o uso da ecocardiografia transesofágica para inserção do eletrodo de marca-passo, a fim de reduzir a radiação sobre o concepto ${ }^{16}$. Gestantes que portam marca-passo artificial deverão ser submetidas ao controle do aparelho, da mesma forma que as não gestantes ${ }^{17}$.

\subsection{3 - Drogas antiarrítmicas (ver cap. 6)}

A difenilhidantoina sódica é contraindicada no primeiro trimestre pelo risco de lábio leporino e fenda palatina e mais raramente a síndrome fenitoínica fetal, que se caracteriza, além das lesões descritas, por má implantação dos ossos do nariz, microcefalia e idiotia.

As demais drogas antiarrítmicas podem ser utilizadas na gestação. A amiodarona é reservada para os casos refratários a outras drogas ou em cardiopatias mais graves, e deve ser evitada em gestantes com disfunção tireoidiana.

O verapamil injetável está indicado no tratamento das taquicardias supraventriculares ${ }^{18}$, assim como a adenosina por via IV rápida ${ }^{19}$.

Em FA crônica com frequência ventricular média elevada, podem ser utilizados digoxina, bloqueador beta-adrenérgico, como propranolol ou metoprolol ${ }^{20}$, ou um bloqueador do cálcio, como verapamil ou diltiazem por via oral ${ }^{18}$.

Gestantes portadoras de prolapso valvar mitral com arritmias sintomáticas devem receber bloqueador beta-adrenérgico (propranolol, metoprolol, atenolol). As com hipertireoidismo 
também se beneficiam com os bloqueadores beta-adrenérgicos, além da medicação específica para a tireoide ${ }^{20}$.

As portadoras de cardiomiopatia dilatada e que apresentam taquicardia ventricular fascicular podem responder bem agudamente ao verapamil injetável (taquicardia ventricular verapamil-responsiva) ${ }^{18}$, lidocaína, procainamida, sotalol, propafenona ${ }^{21,22}$ e amiodarona ${ }^{23}$. Quando há baixo débito é preferível a cardioversão elétrica $^{22}$. Nos casos refratários e recorrentes, indica-se cardioversor-desfibrilador implantável (CDI) ${ }^{22}$.

Quando a taquicardia ventricular é idiopática os bloqueadores beta-adrenérgicos (propranolol, metoprolol) são a primeira escolha ${ }^{22}$.

\section{Resumo das recomendações ${ }^{2,21-26}$}

1) Adotar medidas não farmacológicas para arritmias benignas evitando estimulantes, como café, álcool, estresse, atividade física excessiva, etc (IC).

2) Adotar terapia farmacológica quando os sintomas são intoleráveis e há comprometimento hemodinâmico ou risco materno-fetal (IlaC).

3) A cardioversão elétrica está indicada sempre que a paciente estiver hemodinamicamente instável (IC).

4) Taquicardia atrial por reentrada nodal sem comprometimento hemodinâmico: manobra vagal ou adenosina IV. Caso não reverta, digoxina, betabloqueador ou verapamil, tendo o cuidado de evitar hipotensão. Quando associada à via acessória, preferir procainamida ou quinidina (llbC).

5) Em arritmias ventriculares com estabilidade hemodinâmica, optar por lidocaína ou procainamida. Como profilaxia, preferir betabloqueadores e, quando ineficazes, pode ser optado por sotalol (IC). A amiodarona pode ser utilizada, preferindo doses até $200 \mathrm{mg} /$ dia e monitorando função tireoidiana materna e no neonato. Outra alternativa seria amiodarona em associação com bloqueador betaadrenérgico (IlbC).

6) PCR revertida é indicação de CDI (IC). Para parto cesáreo, é recomendável que o CDI seja desligado para permitir o uso do termocautério.

7) Em bradiarritmia sintomática, pode ser implantado marca-passo com frequência ventricular variável e por via ecocardiográfica (IC).

8) FA aguda geralmente está associada com cardiopatia congênita, doença valvar mitral, hipertireoidismo ou distúrbio eletrolítico. Se houver instabilidade hemodinâmica: cardioversão elétrica (IC). Para cardioversão química, preferir quinidina ou procainamida (IlbC).

9) FA ou flutter crônicos: para controle da frequência ventricular, usar digoxina, bloqueador beta-adrenérgico, diltiazem ou verapamil (IC) e manter com anticoagulante contínuo. Usar uma das heparinas durante toda a gestação ou no primeiro trimestre e duas semanas antes do parto, e anticoagulante oral nos demais períodos (IlbC).

10) Na presença de arritmia ventricular sem cardiopatia estrutural, pensar em hipomagnesemia ou hipocalemia (IB).
18.2 - Ressuscitação cardiorrespiratória básica (RCRB)

Silvia Regina Rios Vieira, Janete Salles Brauner, Citânia L. Tedoldi

É constituído por um conjunto de procedimentos de emergência que consistem no ABCD primário do manejo da parada cardiorrespiratória (PCR).

1) Detectar a inconsciência;

2) Solicitar auxílio e imediatamente iniciar os procedimentos adequados e no solo.

O ABCD primário, cujo algoritmo está representado na figura 1, consta de:

\section{- A}

- Avaliar consciência; se inconsciente:

- Ativar sistema de emergência solicitando desfibrilador.

- Abrir vias aéreas e avaliar respiração; se não houver respiração:

\section{- B}

- Realizar duas ventilações (Breathing) lentas, boca a boca ou boca-válvula-máscara (BVM).

- $\mathbf{C}$

- Avaliar Circulação e se não houver pulso ou sinais de circulação: iniciar compressões torácicas.

- D

- Conectar o Desfibrilador externo automático (DEA) assim que disponível. O DEA pode ser utilizado por qualquer socorrista treinado. Em nível de suporte avançado de vida, pode ser usado imediatamente o monitor/desfibrilador convencional.

As diretrizes internacionais apresentam algumas pequenas diferenças nas recomendações finais e estão disponíveis na Internet: periódico eletrônico Circulation (http://circ. ahajournals.org/content/vol112/22_suppl/) e no site do European Resuscitation Council (http://www.erc.edu/).

Se o socorrista não possui conhecimento das novas recomendações, não é errado realizar ressuscitação conforme diretrizes prévias. As principais alterações promovidas pelo comitê internacional de ressuscitação para a RCRB são:

- As manobras ABCD são iniciadas se a vítima estiver inconsciente e sem respiração normal;

- As ventilações de resgate devem ser realizadas em um segundo (em vez de dois), com volume suficiente para elevar o tórax;

- As compressões torácicas devem ser realizadas numa relação de 30 compressões para duas ventilações.

18.3 - Considerações gerais sobre as manobras de ABCD e o suporte avançado de vida na grávida

1) São dois ou mais pacientes (gestante e feto[s]);

2) A chance de sobrevivência fetal está relacionada com a materna;

3) Na gestação, o consumo de oxigênio está aumentado em $20 \%$ e o transporte de oxigênio está diminuído pela presença da anemia fisiológica. Consequentemente, a hipóxia 


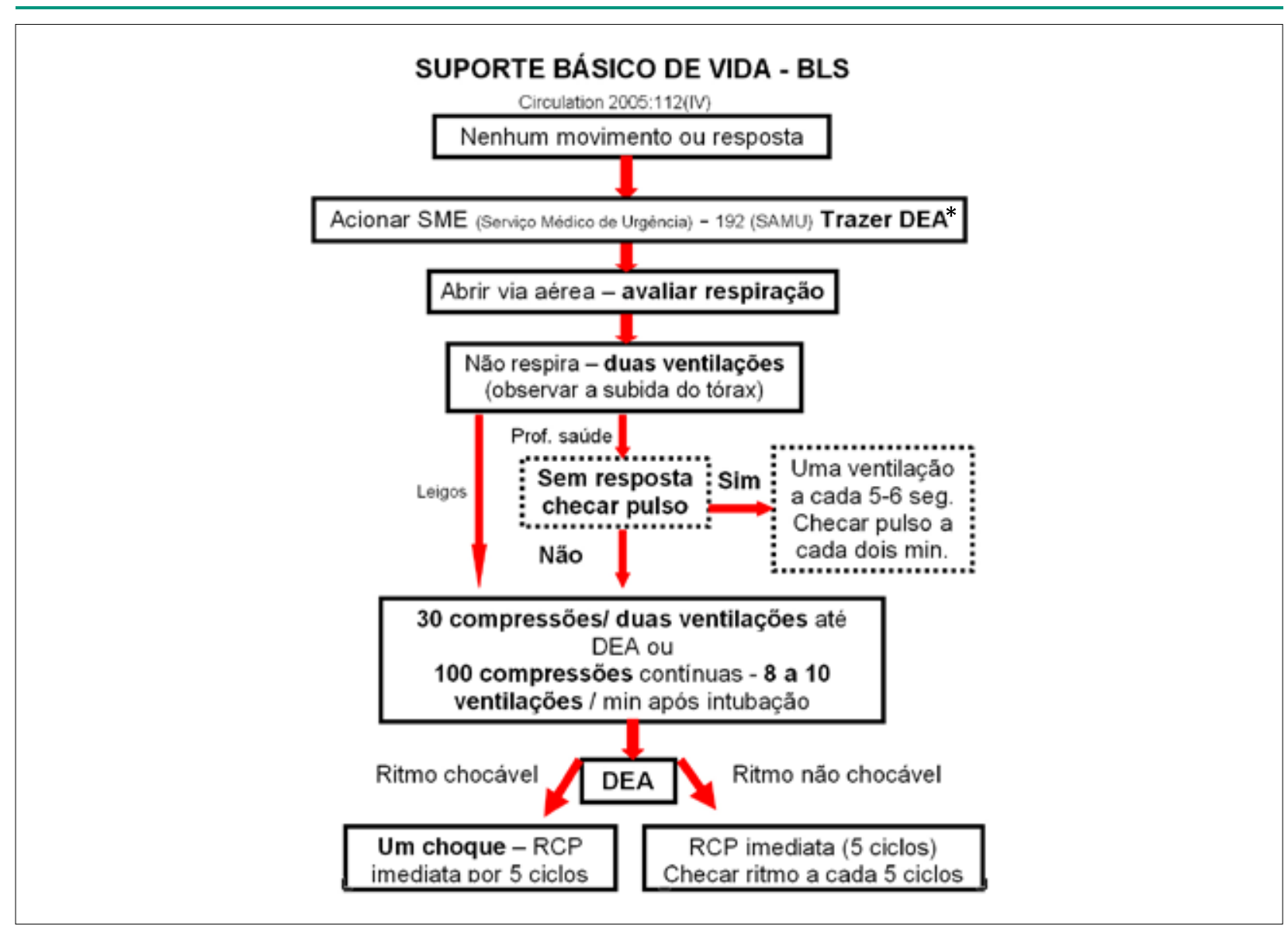

Fig. 1 - Algoritmo do ABCD primário da RCRB. *Desfibrilador externo automático.

ocorre mais rapidamente e com maior comprometimento da oxigenação materna e fetal;

4) O útero gravídico provoca redução do retorno venoso e do débito cardíaco por compressão da cava inferior e da aorta, além da elevação do diafragma;

5) O esfíncter gastroesofágico torna-se insuficiente, mediado por ação hormonal, favorecendo a aspiração de conteúdo gástrico;

6) Algumas condutas dependem da idade gestacional da paciente.

\section{Condutas recomendadas}

1) Via aérea - Manter ventilação mediante compressão da cartilagem cricoide enquanto não for entubada. $\mathrm{Na}$ entubação, geralmente são necessários tubos com calibres menores pela presença de edema. Administrar oxigênio a $100 \%$ e com volumes menores.

2) Circulação - Posicionar paciente em semidecúbito lateral esquerdo e/ou liberar cava e aorta, com afastamento do útero manualmente para a esquerda. As compressões torácicas são realizadas na porção mediana do esterno e as drogas vasopressoras, apesar de reduzirem a circulação uterina, devem ser administradas da mesma forma que em não grávidas.

3) A desfibrilação elétrica não é prejudicial para o feto, mas antes do procedimento deve ser removida a monitorização fetal, se estiver sendo realizada.

4) Devem ser consideradas causas reversíveis como dose excessiva de sulfato de magnésio (usar gluconato de cálcio), embolia pulmonar maciça e AVC isquêmico que podem se beneficiar com fibrinolíticos, infarto agudo do miocárdio que se beneficiaria com reperfusão percutânea, dissecção de aorta, embolia por líquido amniótico, etc.

5) A IG define a necessidade de histerotomia de urgência:

- Se IG < de 20 semanas, não há comprometimento do DC materno pelo volume uterino;

- Entre 20-23 semanas o feto não é viável, mas o esvaziamento uterino pode melhorar o DC materno;

- Se IG $\geq 24$ semanas em hospitais com UTI neonatal após 4-5 minutos de PCR, recebendo manobras adequadas e sem nenhuma resposta, está indicada histerotomia de urgência na tentativa de salvar mãe e feto. 


\section{Referências}

1. Ovando LA, Germiniani H, Miglino R, Cunha GP. Estudo das arritmias cardíacas maternas durante o trabalho de parto e o parto. Arq Bras Cardiol. 1983; 40: 171-6

2. Wolbrette D. Treatment of arrhytnmias during pregnancy. Curr Womens Health Rep. 2003; 3: 135-9.

3. Shotan A, Ostrzega E, Mehra A, Johnson JV, Elkayam U. Incidence of arrhythmias in normal pregnancy and relation to palpitations, dizziness, and syncope. Am J Cardiol. 1997; 79: 1061-4.

4. Leung CY, Brodsky MA. Cardiac arrhythmias and pregnancy. In: Elkayam U, Gleicher N. (editors). Cardiac problems in pregnancy. 3rd ed. New York: Wiley-Liss; 1998. p. 155-75.

5. Avila WS, Rossi EG, Ramires JA, Grinberg M, Bortolotto MR, Zugaib M, et al. Pregnancy in patients with heart disease: experience with 1000 cases. Clin Cardiol. 2003; 26 (3): 135-42.

6. Silversides CK, Harris L, Haberer K, Sermer M, Colman JM, Siu SC. Recurrence rates of arrhythmias during pregnancy in women with previous tachyarrhythmia and impact on fetal and neonatal outcomes. Am J Cardiol. 2006; 97: 1206-12

7. Tawam M, Levine J, Mendelson M, Goldberger J, Dyer A, Kadish A. Effect of pregnancy on paroxysmal supraventricular tachycardia. Am J Cardiol. 1993; 72: 838 .

8. Lee $\mathrm{SH}$, Chen SA, Wu TJ, Chiang CE, Cheng CC, Tai CT. Effects of pregnancy on first onset and symptoms of paroxysmal supraventricular tachycardia. Am J Cardiol. 1995; 76: 675-8.

9. Penkala M, Hancock EW. Wide-complex tachycardia in pregnancy. Hosp Pract. $1993 ; 24: 63-4$

10. Brodsky M, Doria R, Allen B, Sato D, Thomas G, Sada M. New-onset ventricular tachycardia during pregnancy. Am Heart J. 1992; 123: 933-4.

11. Widerhorn JK, Widerhorn AM, Rahimtoola SH, Elkayam U. WPW syndrome during pregnancy: increased incidence of supraventricular arrhythmias. Am Heart J. 1992; 124: 796-8.

12. Doig JC, McComb JM, Reid DC. Incessant atrial tachycardia accelerated by pregnancy. Br Heart J. 1992; 67: 266-8.

13. Seth R, Moss AJ, McNitt S, Zareba W, Andrews ML, Qi M, et al. Long QT syndrome and pregnancy. J Am Coll Cardiol. 2007; 49: 1092-8.

14. Dalvi BV, Chauduri A, Kulkarni HL, Kale PA. Therapeutics guidelines for congenital complete heart block presenting in pregnancy. Obstet Gynecol. 1992; 79: 802-4.

15. Ramsewak S, Persad P, Perkins S, Narayansinger GV. Twin pregnancy in a patient with complete heart block. Clin Exp Obstet Gynecol. 1992; 19: 166-7.

16. Jordaens LJ, Vandenbogaerde JF, Van De Bruaene P, Buyzere MD Transesophageal echocardiography for insertion of a physiological pacemaker in early pregnancy. Pacing Clin Electrophysiol. 1990; 13: 955-7.

17. Terhaar M, Schakenbach L. Care of the pregnant patient with a pacemaker. J Perinatol Neonatol Nurs. 1991; 5: 1-12.

18. Germiniani H. Verapamil. In: Germiniani H. (editor). Diagnóstico e terapêutica das arritmias cardíacas. 3aㅡ ed. Rio de Janeiro: Guanabara; 1990. p. 303-5.

19. Elkayam U, Goodwin TM. Adenosine therapy for supraventricular tachycardia during pregnancy. Am J Cardiol. 1995; 75: 521-3.

20. Hurst AK, Hoffman K, Frishman WH, Elkayam U. The use of beta-adrenergic blocking agents in pregnancy and lactation. In: Elkayam U, Gleicher N. (editors). Cardiac problems in pregnancy. 3rd ed. New York: Wiley-Liss; 1998 p. 419-33.

21. Chow T, Galvin J, McGovern B. Antiarrhythmic drug therapy in pregnancy and lactation. Am J Cardiol. 1998; 82: 581-621.

22. Gowda RM, Khan IA, Mehta NJ, Vasavada BC, Sacchi TJ. Cardiac arrhythmias in pregnancy: clinical and therapeutic considerations. Int J Cardiol. 2003; 88: 129-33.

23. Matsumura LK, Born D, Kunii IS, Franco DB, Maciel RM. Outcome of thyroid function in newborns from mothers treated with amiodarone. Thyroid. 1992; 2: $279-81$

24. Tan HL, Lie KI. Treatment of tachyarrhythmias during pregnancy and lactation Eur Heart J. 2001; 22: 458-64.

25. ACC/AHA/ESC 2006 guidelines for management of patients with ventricula arrhythmias and the prevention of sudden cardiac death. Eur Heart J. 2006; 27: 2099-140.

26. ACC/AHA/ESC 2006 Guidelines for management of patients with atrial fibrillation. Circulation. 2006; 114: 700-52.

27. 2005 American HeartAssociation Guidelines for Cardiopulmonary Resuscitation and Emergency Cardiovascular Care. ECC Committee Subcommittee and Task Forces of the AHA. Circulation. 2005; 112: (24 Suppl.): IV1-203.

\section{9 - Anestesia e analgesia de parto \\ Sérgio Renato Guimarães Schmidt, André Prato Schmidt, Adriana Prato Schmidt}

\section{1 - Introdução}

A grande aceitação e utilização das técnicas de anestesia regional tornou a anestesia obstétrica uma das principais e mais difundidas práticas anestésicas ${ }^{1}$.

No cuidado anestésico de uma gestante, os dois pacientes (a mãe e o feto) sofrem as consequências da conduta. É necessário um profundo conhecimento das alterações fisiológicas que acompanham a gestação e de suas interações com a modalidade anestésica escolhida. Falhas em considerar tais fatores podem gerar consequências catastróficas para ambos ${ }^{1}$.

19.2 - Alterações fisiológicas na gestação e a anestesia obstétrica

A gestação provoca alterações nas funções de todo o organismo, que podem ser: respiratórias (aumento do consumo de oxigênio, do volume ventilatório, da frequência ventilatória e da pressão parcial de oxigênio, com diminuição proporcional da pressão parcial de gás carbônico); cardiovasculares (aumento do volume sanguíneo e plasmático, do débito cardíaco, frequência cardíaca e diminuição da resistência vascular periférica); hematológicas (diminuição de hemoglobina e plaquetas e aumento dos fatores de coagulação); e renais (aumento da taxa de filtração glomerular) ${ }^{1,3}$. Também provoca outras alterações fisiológicas aqui enfatizadas devido a sua potencial interação com o procedimento anestésico: obstrução da veia cava inferior por um útero aumentado provocando ingurgitamento do plexo venoso epidural; síndrome da hipotensão supina (palidez, hipotensão, sudorese, náuseas e vômitos) em $20 \%$ dos casos; redução da motilidade gástrica e tônus do esfincter esofágico inferior e aumento da secreção gástrica, predispondo a alto risco de regurgitação; sensibilidade aumentada aos anestésicos locais durante anestesia regional e diminuição 